\title{
Über Schutzimpfung gegen Pest auf Formosa.
}

\author{
Von
}

Dr. K. Tsukiyama,

Direktor des staatlichen Kilung-Hospitals auf Formosa (Japan).

Seitdem Haffkine ${ }^{1}$ im Jahre 1897 in Ostindien zwecks des Pestschutzes beim Menschen ein aktives Immunisierungsverfahren eingeführt hatte, wurde auch ron der deutschen Pestrommission und von LustigGaleotti die Schutzimpfung gegen Pest empfohlen.

Wenn auch eine endgültige Beurteilung der gewonneǹen Resultate bisher noch nicht möglich ist; so wird doch anerkannt, daB die Schutzimpfung als Vorbeugungsmittel gegen die Pest nicht mehr zu entbehren ist. Es ist also notwendig, daB beim Ausbruch der Pest diese Schutzimpfung bei allen Personen ausgeführt wird, die direkt der Infektionsgefahr ausgesetzt sind, so namentlich bei Ärzten, dem Wartepersonal von Pestkranken, bei Dienern in Pestuntersuchungsinstituten und bei Desinfekteuren, ferner bei Bew.ohnern kleinerer Gemeinwesen, bei Mannschaften auf Schiffen und in Kasernen.

In Japan selbst wurde im Jahre 1899 von Prof. Dr. Kitasato, Direktor des Regierungsinstituts für Infektionskrankheiten in Tokio, ein besonderer Impfstoff hergestellt, mit dem von Prof. Dr. Shiga, Prof. Dr. Shibayama (beide Sektionschef des obengenannten Institutes) und Dr. Hada in Osaka, Kobe und Wakayama praktische Versuche angestellt wurden. Auch von Prof. Dr. Jokote wurde die Schutzimpfung einer Prüfung unterworfen.

${ }^{3}$ Kolle u. Wasserma n n, Handbuch der pathogenen Mikroorganismen. Bd.II. T. II. S. 932 ff. Jena 1904.

Zeitschr. f. Hygiene. LVIII. 
Die Ergebnisse dieser aktiven Schutzimpfung, die sowohl in Indien wie in Japan vorgenommen wurde, sind im wesentlichen folgende:

In dem Byculla-Gefängnis zu Bombay erkrankten innerhalb vier Jahren (1897 bis 1901) von 183 Nichtgeimpften 12, davon starben 6. Dagegen erkrankten von 154 unter denselben Verhältnissen lebenden geimpften Personen nur 2.

In dem portugiesischen Damaun wurden vom 23. bis 26. März 1897 1017 Personen geimpft, davon erkrankten bis zum 23. April 23 und starben 6 ( $=$ einer Sterblichkeit von 0.58 Prozent). Von den $7213 \mathrm{Un}$ geimpften starben in demselben Zeitraum 716 (= einer Sterblichkeit von 9.9 Prozent). Von 1639 Personen, geimpft vom 17. April bis 2. Mai, erkrankten rom 2. bis 19. Mai 64, es starben davon $27=1.6$ Prozent. Von den nicht geimpften Personen sind in der gleichen Zeit 5869 Krankheitsfalle mit 674 Todesfällen ( $=11.5$ Prozent) zu verzeichnen. Ferner wurden vom 21. bis 23. Mai 364 Personen der Schutzimpfung unterworfen; davon erkrankten bis Ende Mai 4 und starben $3(=0.14$ Prozent). Von den Ungeimpften erkrankten 4643 und starben 93 (=2.0 Prozent).

In Lanowli erkrankten von 377 Ungeimpften 78 und starben 57 , von 323 Geimpften dagegen erkrankten nur 14 und starben 7; der Unterschied in der Zahl der Todesfälle beträgt also 85.7 Prozent.

In Artillerie-Kantonnement zu Kirkee kamen unter 671 Geimpften 32 Eirkrankungen (4.7 Prozent) und 17 Todesfälle (2.5 Prozent) vor, unter den 859 Ungeimpften dagegen 143 Erkrankungen (16.6 Prozent) und 98 Todesfälle (11.4 Prozent).

In dem Umerkadi-Gefängnis zu Bombay erkrankten unter $147 \mathrm{Ge}$ impften 3, unter 127 Ungeimpften 10, davon starben 6 .

In Undhera fand man bei 71 Geimpften 8 Krankheitsfälle und 3 Todesfälle und bei 64 Ungeimpften 27 Krankheitsfälle und 26 Sterbefälle, also unter den Geimpften 89.6 Prozent weniger Mlortalität.

In Hublini verminderte sich auch bedeutend die Zahl der Todesfälle dureh Impfung.

In Japan war weder ein Erkrankungsfall noch ein Todesfall unter den Geimpften der obengenannten Städte zu rerzeichnen.

Neben der Wirksamkeit dieses Impfstoffes muß noch konstatiert werden, wie lange die durch die Impfung erlangte Immunität andauert? Die Antwort auf diese Frage ist je nach den Autoren verschieden:

Nach Haffkine wird die Immunitätsdauer auf wenigstens eine Epidemieperiode ( 4 bis 6 Monate) festgesetzt. ${ }^{1}$

1 Lancet. 1899. 
Die deutsche Pestuntersuchungskommission fand, daB die Immunität 7 Tage nach der Impfung auftritt und 5 bis 6 Monate dauert. ${ }^{1}$

Calmette berichtet über die Ergebnisse der Schutzimpfung mit Haffkines Impfstoff bei Affen, Meerschweinchen und Mäusen. Danach wurden von einer 1 Monat alten Bouillonkultur, die durch einstündiges Erwärmen auf $70^{\circ}$ abgetötet war, 3 com auf einmal den genannten Tieren injiziert. Nach 7 Tagen wurde erst der Beginn der Immunität erkennbar und dieselbe dauerte bei Meersehweinchen 3 Wochen, bei Affen 1 Monat, und bei Mäusen hielt sie sogar durch die einmalige Impfung mit 2 ocm 3 Monate an. Zwar können diese Versuchsergebnisse nicht ohne weiteres auf die Immunität beim Menschen übertragen werden. Dennoch darf man danach annehmen, daB beim Menschen, der sehr empfänglich für das Pestgift ist, die Immunität von längerer Dauer sein wird, analog den Versuchsergebnissen bei Mäusen, die ebenfalls sehr empfänglich für Pest sind und bei denen die Immunität viel länger anhält, wie bei den weniger empfänglichen Meerschweinchen. ${ }^{2}$

Prof. Dr. Shibayama und Dr. Hada (Japan) haben folgenden Bericht über den Impfrersuch mit Kitasa tos Impfstoff bei Meerschweinchen erstattet:

Es ist sicher, daB die Versuchstiere nach der Impfung mit Impfstoff unter bestimmten Bedingungen Widerstand gegen die Pestinfektion zeigen und dadurch dem Tode entgehen und länger am Leben bleiben. Betreffs der Dauer der vorbeugenden Wirkung können zwar die beiden Herren zuverlässige Resultate nicht aufstellen, denn sie muBten wegen anderweitiger Arbeiten die Untersuchungen zeitweise einstellen. Aber so viel sei festgestellt, daB die Immunität beinahe 3 Monate anhalten könne, ja sogar nach 4 Monaten dieselbe mehr oder weniger vorhanden sein könne. Sie behaupten ferner, daB die Immunität erst 10 Tage nach der Impfung auftritt. $^{3}$

Wie oben angeführt ist, kann die Immunitätsdauer nicht einheitlich festgestellt werden. Es unterliegt aber keinem Zweifel, daß die Ansichten über die präventive Wirkung der seit etwa $10 \mathrm{Jahren}$ ins Leben gerufenen Schutzimpfung gegen Pest mannigfaltig sind, wie auch bei der Pockenlymphe, die seit 100 Jahren überall verbreitet ist, die Immunitätsdauer noch nicht einheitlich festbestimmt ist.

1 Centralblatt für Bakteriologie. Bd. XXIII. Nr. 16/17.

Zeitschrift für Bakteriologie. (Japan.) Nr. 58.

A. a. 0. Nr. 65 . 
Was die Pestepidemie in Formosa speziell anbelangt, so ist es hier eigentümlich, daß in jedem zweiten Jahre seit der Annektion durch Japan (1895) große Epidemien geherrscht haben, während in anderen Jahren nur sporadische Pestfälle vorkamen; so trat die Pestinfektion in den Jahren 1897, 1899 epidemisch und in den Jahren 1896, 1898, 1900 sporadisch auf.

Eine genaue Erörterung über alle diese Epidemiejahre kann nicht gegeben werden, denn die vorliegende Abhandlung beschäftigt sich nur mit den Resultaten der Schutzimpfung, welche erst in den beiden letzten Epidemiejahren (1901 und 1904) ausgeführt worden ist.

Aus dieser regelmäBig wiederkehrenden Epidemieperiode, zumal aus dem Vorhandensein der massenhaft gestorbenen Ratten, vom Winter 1900 bis Frühling des folgenden Jahres in Tainan sah man voraus, daB in diesem Jahre (1901) wieder eine Epidemie herrschen würde. Wie vermutet kamen schon am 29. Norember 1900 im Zollamt Anping ein Fall und am 25. Dezember im Gefängnis auch ein Fall vor. Seit dieser Zeit verbreitete sich die Pest mit groBer Geschwindigkeit in der Umgebung, so daB rom Januar bis Mitte April 1901541 Pestfälle in dem Regierungsbezirk von Tainan gezählt wurden.

Zur Zeit dieser Epidemie hatte das Gouvernement von Tainan eine gründliche desinfizierende. Reinigung der Straßen und Wohnungen und zugleich die Schutzimpfung mit Kitasatos Impfstoff energisch empfohlen. Der Verfasser dieser Abhandlung beschäftigte sich hauptsächlich mit der Schutzimpfung. Zu diesem Zwecke wurden folgende MaBregeln getroffen:

1. In den Epidemiegegenden muB nicht bloB bei den Leuten in dem Hause, wo der Erkrankte wohnt, sondern auch bei der ganzen Einwohnerschaft in der Umgebung die Schutzimpfung ausgeführt werden.

2. Die sämtlichen Beamten der Regierung und Privatkorporationen der Epidemiegegend müssen unbedingt geimpft werden.

3. AuBerdem trönnen die Leute, die aus eigenem Antrieb die Schutzimpfung wünschen, zu jeder Zeit geimpft werden.

Zur Impfung wurde der konzentrierte Impfstoff gebraucht. Das Quantum dieses Impfstoffs wurde mit Rücksicht auf die körperliche Beschaffenheit und das Alter genau der von dem Institut für Infektionskrankheiten in Tokio vorgeschriebenen Gebrauchsanweisung entsprechend differenziert.

Für die Anwendung des Impfstoffs wurden folgende Regeln festgesetzt:

1. Die Impfung soll bei jeder Person zweimal ausgeführt werden.

2. Die Menge des Impfstoffs ist folgendermaßen bemessen; sie kann aber nach Körperbeschaffenheit nach oben und nach unten einige $A b-$ weichungen erleiden. a) Bei der ersten Impfung soll die Menge nach 
dem Lebensalter bestimmt werden, und zwar bei den Impflingen von 16 bis 50 Jahren $1.0 \mathrm{~cm}$, bei solchen von 11 bis 15 und über 50 Jahren $0.7 \mathrm{com}$, und bei den 6 bis 10 jährigen $0.5 \mathrm{com}$. Es kann auch bei den 5 bis $1 / 2$ jährigen Kindern die Impfung vorgenommen werden. In diesem Falle nehme man je nach dem Alter nicht über $0.3^{\mathrm{cm}}$ Impfstoff. b) Bei der zweiten Impfung soll je nach dem Grad der Reaktion 2 bis 3 fach mehr als die Menge bei der ersten Impfung genommen werden.

3. Die zweite Impfung soll 5 bis 10 Tage nach der ersten Impfung ausgeführt werden.

4. Zur Impfung wähle man die weichen Hautteile der Subskapulargegend, halte die Impfnadel schräg und steche tief unter die Haut. Es kann auch je nach dem Beruf in andere Körperteile geimpft werden. Die zu impfende Gegend soll vor und nach der Ausfübrung mit Alkohol sorgfältig gereinigt werden. Die Impfnadel soll nach dem Gebrauch mit 5 prozentigem und nachher nochmals mit 0.5 prozentigem Karbolwasser gründlich desinfiziert werden.

5. Der Impfstoff soll vor dem Gebrauch jedesmal geschüttelt werden.

6. Der Impfstoff soll im kalten und dunklen Raum aufbewahrt werden; derselbe soll über zwei Monate nach der Herstellung nicht verwendet werden.

7. Hat der Behälter das Zeichen „,konzentriert“, so nehme man von dessen Inhalt die Hälfte des oben genannten Quantums.

Der Verfasser hat von August 1900 bis Februar 1901 regelmäBig zweimal geimpft, von da an aber erschienen die erst Geimpften nicht zur zweiten Impfung, da die Geimpften sich wegen der reaktiven Erscheinungen (Kopfweh, Fieber usw.) nach der ersten Impfung fürchteten, nochmals geimpft zu werden. Aus diesem Grunde wurde seit Februar 1901 ein solches Quantum auf einmal geimpft, wie es für zweimalige Impfungen gelten soll.

Das Resultat der Impfung konnte der Verfasser wegen der angehäuften Arbeit nicht bei allen Geimpften konstatieren. So viel ist aber sicher, daB bei den 1358 geimpften Beamten und anderen Korporationsmitgliedern, die er selbst genau betrachtet hat, eines oder mehrere von nachstehenden Symptomen als Reaktionserscheinungen zu beobachten waren: Müdigkeit, Kopfschwere, Kopfweh, Temperatursteigerung, lokaler Schmerz, selten Anschwellung der Hals- und Achseldrüsen. Die reaktionslos Gebliebenen betrugen 3 Prozent von den beobachteten Geimpften.

Zur übersichtlichen Darstellung der Ergebnisse der Schutzimpfung gegen Pest diene Tabelle I. Die Impfung seit August 1900 ist, wenn auch damals die Pest nicht epidemisch auftrat, in der Tabelle mitgezählt. 


\begin{tabular}{|c|c|c|c|c|c|c|c|c|c|c|c|c|c|c|c|c|}
\hline$\Rightarrow$ & क & $\vec{v}$ & 穴 & $e_{00}$ & $\overrightarrow{10}_{0}$ & $\Xi$ & $\vec{b}$ & $\bullet$ & $\infty$ & -1 & $\sigma$ & or & 1 & అు & $N$ & - \\
\hline$\approx$ & 苍 & $=$ & 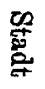 & 㣢 & 蛋 & 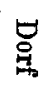 & 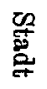 & $\approx$ & $\approx$ & 导 & $\approx$ & 蛋 & 봉 & $=$ & $=$ & 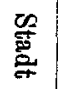 \\
\hline 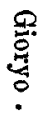 & 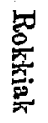 & 惫 & 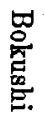 & 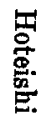 & 占. & & 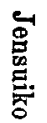 & 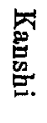 & 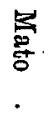 & $\begin{array}{l}\text { OD } \\
\text { O्I } \\
\text { E्g } \\
.\end{array}$ & 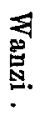 & 宽 & 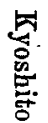 & 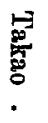 & 胥 & 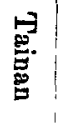 \\
\hline$\cdot$ & 馜 & $\cdot$ & $\cdot$ & $\cdot$ & . & . & . & . & $\therefore$ & $\cdot$ & . & $\cdot$ & $\cdot$ & $\bullet$ & . & . \\
\hline$\cdot$ & $D$ & - & - & $\cdot$ & - & $\cdot$ & • & - & . & $\cdot$ & $\cdot$ & $\cdot$ & $\cdot$ & $\cdot$ & . & $\cdot$ \\
\hline . & . & . & . & . &. &. & . &. &. & 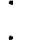 & 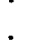 & 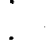 &. &. &. & 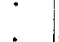 \\
\hline$\stackrel{5}{0}$ & $\stackrel{\mathbb{D}}{\mathscr{D}}$ & 怘 & $\underset{⿱ 中}{\stackrel{m}{*}}$ & 蚍 & 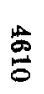 & 芯 & 蝑 & 岕 & 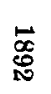 & $\underset{\mathbb{A}}{\mathbb{N}}$ & : & $\stackrel{0}{\circ}$ & g' & $\begin{array}{l}\infty \\
\stackrel{0}{0}\end{array}$ & $\underset{N}{\mathbb{N}}$ & $\begin{array}{l}\overrightarrow{8} \\
\underset{\sim}{*}\end{array}$ \\
\hline$\stackrel{-0}{\varrho}$ & $\begin{array}{l}\vec{G} \\
\vec{J}\end{array}$ & 总 & 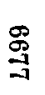 & 怘 & $\begin{array}{l}\overrightarrow{0} \\
\ddot{\leftrightarrow}\end{array}$ & $\begin{array}{l}\stackrel{c}{\leftrightarrows} \\
\stackrel{g}{\sigma}\end{array}$ & 总 & 苋 & 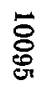 & $\vec{\infty}$ & 蛋 & 㤩 & $\stackrel{\mathscr{\infty}}{\mathscr{\infty}}$ & 悹 & 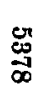 & $\begin{array}{l}\mathbb{A} \\
\text { 焉 } \\
\text { D. }\end{array}$ \\
\hline
\end{tabular}

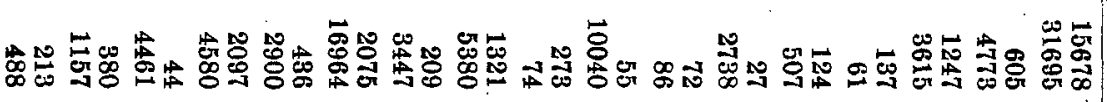

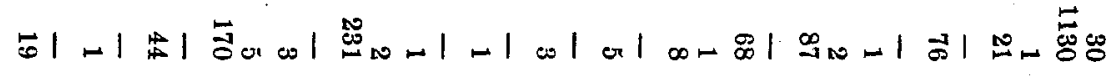

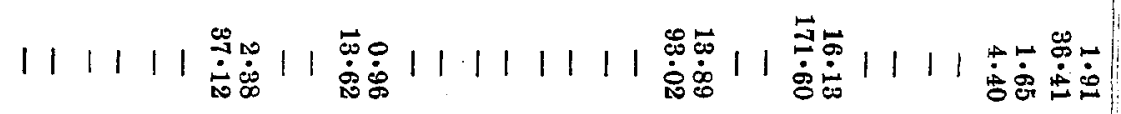

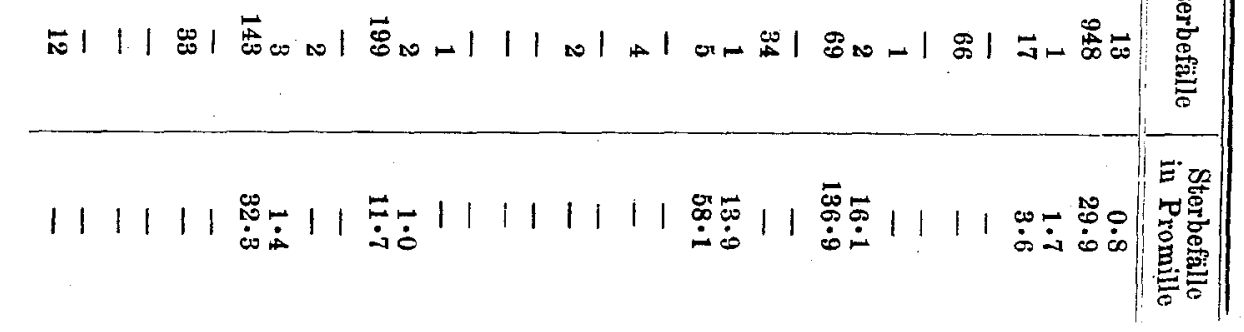


Um die Zahl der Krankheitsfälle und Sterbefälle bei Geimpften und Ungeimpften genau zu vergleichen, werden die Krankheits- und Sterbefälle ausschlieBlich bei den Geimpften von der Tabelle I herausgenommen und in der Tabelle II zusammengestellt.

Tabelle II.

Vergleichende Zusammenstellung der Krankheits- und Sterbefälle bei Ungeimpften und Geimpften.

\begin{tabular}{|c|c|c|c|c|c|c|c|}
\hline \multirow{2}{*}{ 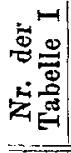 } & \multirow{2}{*}{$\begin{array}{c}\text { Ort- } \\
\text { schaften }\end{array}$} & \multicolumn{3}{|c|}{ A. Krankheitsfälle in \% \% } & \multicolumn{3}{|c|}{ B. Sterbefalle in $\%$} \\
\hline & & $\begin{array}{c}\text { Un- } \\
\text { geimpfte }\end{array}$ & Geimpfte & $\begin{array}{c}\text { weniger } \\
\text { bei } \\
\text { Geimpften }\end{array}$ & $\begin{array}{c}\text { Un- } \\
\text { geimpfte }\end{array}$ & Geimpfte & $\begin{array}{c}\text { weniger } \\
\text { bei } \\
\text { Geimpften }\end{array}$ \\
\hline 1 & Tainan & $36 \cdot 41$ & $1 \cdot 91$ & $34 \cdot 5$ & $29 \cdot 88$ & 0.82 & 29.7 \\
\hline 2 & Anping & $4 \cdot 40$ & $1 \cdot 65$ & $2 \cdot 8$ & $3 \cdot 56$ & 1.65 & $1 \cdot 9$ \\
\hline 5 & Taikao & $171 \cdot 60$ & $16 \cdot 13$ & $155 \cdot 5$ & $136 \cdot 09$ & $16 \cdot 13$ & $120 \cdot 0$ \\
\hline 6 & Sobun & 93.02 & $13 \cdot 89$ & $79 \cdot 1$ & $58 \cdot 14$ & $13 \cdot 89$ & $44 \cdot 3$ \\
\hline 12 & Kagi & $13 \cdot 62$ & 0.96 & $12 \cdot 7$ & $11 \cdot 73$ & $0 \cdot 96$ & $10 \cdot 8$ \\
\hline 14 & Bokushi & $37 \cdot 12$ & $2 \cdot 38$ & $34 \cdot 7$ & $32 \cdot 32$ & $1 \cdot 43$ & $30 \cdot 9$ \\
\hline
\end{tabular}

Wenn diese einzelnen Fälle nicht in Prozenten, sondern zahlenmäßig zusammengestellt werden, so bekommt man folgendes Bild:

Tabelle III.

\begin{tabular}{|c|c|c|c|}
\hline & Krankheitsfälle & Sterbefälle & Prozente \\
\hline Geimpfte . . . . . . & 41 & 22 & $53 \cdot 7$ \\
\hline Ungeimpfte $\cdot \cdot \cdot \cdot \cdot$ & 1511 & 1265 & $83 \cdot 7$ \\
\hline
\end{tabular}

Hieraus ist $\mathrm{zu}$ ersehen, daB die Krankheitsfälle bei Geimpften weniger als den 36. Teil wie von den Ungeimpften betragen und daB sich die Sterbefalle bei ersteren um etwa 30 Prozent vermindert haben.

Diese bedeutende Differenz der Krankheits- und Sterbefälle zwischen den Geimpften und Ungeimpften, wie sie die Tabellen II und III zeigen, ist auf die Wirkung der Schutzimpfung zurückzuführen.

Bei diesen 41 Krankheitsfällen der Geimpften ist ferner zu untersuchen, wie lang die Zeitdauer von der Impfung bis zum Beginn der Krankheit ist und in welchem Zeitabschnitt, in dem die Krankheit auftritt, die erkrankten Fälle die meisten Sterbefälle zeigen. Dazu wird die Tabelle IV zusammengestellt. 
Tabelle IV.

\begin{tabular}{|c|c|c|c|c|c|}
\hline Geimpfte & \multicolumn{2}{|c|}{ Tage nach Impfung } & $\begin{array}{l}\text { Krankheits- } \\
\text { fälle }\end{array}$ & Sterbefälle & $\begin{array}{c}\text { Prozentzahl } \\
\text { der Erkrankten }\end{array}$ \\
\hline \multirow{7}{*}{20455} & \multicolumn{2}{|c|}{ · innerhalb 10 Tagen } & 6 & 2 & 0.03 \\
\hline & \multicolumn{2}{|c|}{ zwischen $10-30$ Tagen } & 12 & 7 & 0.05 \\
\hline & \multirow{2}{*}{\multicolumn{2}{|c|}{$\begin{array}{ll}" & 31-60 \quad " \\
& 61-90 \quad "\end{array}$}} & 12 & 6 & 0.05 \\
\hline & & & 4 & 3 & 0.02 \\
\hline & & $91-120$ & 4 & 2 & 0.02 \\
\hline & " & $121-150$ & 1 & 0 & 0.004 \\
\hline & über & 150 & 2 & 2 & 0.004 \\
\hline
\end{tabular}

Aus dieser Tabelle ergibt sich, daß in der Zeit vom 30. bis 60. Tag nach der Impfung sowohl die Krankheitsfälle, wie auch Sterbefälle am häufigsten auftreten.

AuBerdem ist noch merkwürdig, daB, wie in der Tabelle I angeführt ist, in den 11 Orten (Nr. 3, 4, 6, 8, 9, 10, 11, 13, 15, 16, 17) bei Geimpften gar keine Krankheitsfälle zu verzeichnen sind. Insbesondere sind in den Orten Nr. 8, 11, 15, wo diejenigen Leute geimpft wurden, welche von Pestkranken umgeben waren oder direkt in Berührung kamen, keine Pestkranken $z u$ finden.

Die bis jetzt gegebene Darstellung betrifft die Schutzimpfung sowohl bei den Einheimischen ${ }^{1}$ als auch bei den von Japan (Mutterland) Eingewanderten. $\mathrm{Da}$ aber die Lebensweise, Pflege und Behandlung der Krankheit der Einheimischen von den Eingewanderten wesentlich verschieden sind, so bedarf es einer weiteren Untersuchung für diejenigen Menschen, die eine echt japanische Lebensweise usw. führen (nicht einheimisch formosaische). Zum Zwecke dieser Untersuchung wurden die Beamten, Mitglieder verschiedener Gesellschaften und Vereine und Schüler, welche in einer einheitlichen, dem Mutterland entsprechenden, Weise und in den anderen gleichen Verhältnissen leben, in einer Tabelle zusammengefaßt. Unter den genannten Beamten und Gesellschaftlern nehmen 90 Prozent Japaner ein, während die anderen 10. Prozent zwar Einheimische (meistens Unterbeamte, Diener und andere Untergeordnete) sind, aber dieselbe Lebensweise usw. wie die Japaner haben. In den Schulen (Nr. 10, 11, 16, 19, 20, 21, 22 in der Tabelle V) sind außer Lehrer und Beamten ausschließlich Einheimische,. welche unter strengster Aufsicht und Pflege die mutterländische Lebensweise führen.

${ }^{1}$ Hier versteht man unter Einheimischen diejenigen Einwohner, welche von jeher, vor Annektion von Formosa durch Japan, dort leben und chinesischen Ursprungs sind. 
Tabelle V.

Resultate der Schutzimpfung bei Beamten der Regierung und der anderen Korporationen einschlieBlich Schüler in Tainan und anderen Städten.

\begin{tabular}{|c|c|c|c|c|c|c|}
\hline $\mathrm{N}$ a $\mathrm{m} \mathrm{e}$ & Person & $\begin{array}{c}\text { Geimpfte } \\
\text { Ùngeimpft. }\end{array}$ & 商 & 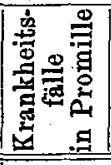 & 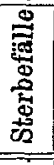 & 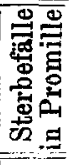 \\
\hline Zollamt in Anping..... & 218 & $\begin{array}{r}217 \\
1\end{array}$ & $\overrightarrow{1}$ & $1000 \cdot 0$ & $\overline{-}$ & $\overline{-}$ \\
\hline Zweigamt in Anping $\vee$. Tainan Verwaltung & 45 & $\begin{array}{l}35 \\
10\end{array}$ & $\begin{array}{l}1 \\
3\end{array}$ & $\begin{array}{r}2.8 \\
300 \cdot 0\end{array}$ & 1 & - \\
\hline Hospital in Tainan & 130 & 130 & - & $\overline{-}$ & $\overline{-}$ & $\overline{-}$ \\
\hline Gefängnis in Tainan & 699 & $\begin{array}{r}696 \\
3\end{array}$ & $\overline{3}$ & $\overline{1000} \cdot 0$ & $\overline{2}$ & $\overline{-}$ \\
\hline Landgericht in Tainan . . & 74 & $\begin{array}{r}\mathbf{7 2} \\
\mathbf{2}\end{array}$ & $\begin{array}{l}1 \\
2\end{array}$ & $\begin{array}{r}13 \cdot 9 \\
1000 \cdot 0\end{array}$ & $\overline{2}$ & $\overline{-}$ \\
\hline Verwaltung in Tainan . . . . & 303 & $\stackrel{303}{-}$ & $\overline{-}$ & - & - & - \\
\hline Landesamt in Tainan $\cdot \cdot$ & 343 & $\begin{array}{r}342 \\
1\end{array}$ & $\begin{array}{l}1 \\
1\end{array}$ & $\begin{array}{r}2 \cdot 9 \\
1000 \cdot 0\end{array}$ & $\overline{1}$ & - \\
\hline eminar in Tainan.... & 138 & $\begin{array}{r}114 \\
24\end{array}$ & $\underline{-}$ & - & $\overline{-}$ & $\overline{-}$ \\
\hline Volksschule in Tainan. & 204 & $\begin{array}{r}164 \\
40\end{array}$ & $\overline{1}$ & $\overline{25.0}$ & 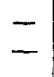 & - \\
\hline I. Volksschule für Einheimische & 161 & $\begin{array}{r}49 \\
112\end{array}$ & $\overline{-}$ & $\underline{-}$ & - & $\overline{-}$ \\
\hline 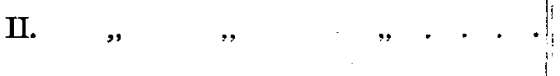 & 71 & $\begin{array}{l}56 \\
15\end{array}$ & $\overline{-}$ & - & - & - \\
\hline Postamt in Tainan . . . . . & 230 & 230 & - & $\overline{-}$ & - & $\overline{-}$ \\
\hline Waffenbureau in Tainan . & 42 & $\begin{array}{r}7 \\
35\end{array}$ & $\overline{-}$ & $\overline{-}$ & $=$ & $\overline{-}$ \\
\hline Observatorium in Tainau. & 10 & $\begin{array}{l}8 \\
2\end{array}$ & - & $\overline{-}$ & $\overline{-}$ & $\overline{-}$ \\
\hline dzamt in Tainan & 11 & $\begin{array}{l}9 \\
2\end{array}$ & - & - & $\because$ & 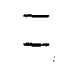 \\
\hline Armenhaus in Tainan & 66 & $\begin{array}{r}65 \\
1\end{array}$ & $\overline{1}$ & $10 \overline{-} \cdot 0$ & $\overline{-}$ & $\overline{-}$ \\
\hline Bahnhof in Tainan . . & 77 & $\begin{array}{l}26 \\
51\end{array}$ & $\overline{-}$ & - & $\overline{-}$ & $\overline{-}$ \\
\hline Infektionskrankenhaus in Tainan . . & 25 & $\begin{array}{r}20 \\
5\end{array}$ & $\overline{2}$ & $4 \overline{00} \cdot 0$ & $\overline{2}$ & $\overline{-}$ \\
\hline Websehule in Tainan . & 61 & 61 & $\overline{-}$ & - & - & $\overline{-}$ \\
\hline Volksschule für Einheimische in Kagi & 217 & $\begin{array}{r}130 \\
87\end{array}$ & $\begin{array}{r}2 \\
11\end{array}$ & $\begin{array}{r}15 \cdot 3 \\
126 \cdot 4\end{array}$ & $\begin{array}{l}2 \\
7\end{array}$ & $\begin{array}{l}15 \cdot 4 \\
80 \cdot 5\end{array}$ \\
\hline Bokushi & 176 & $\begin{array}{r}55 \\
121\end{array}$ & $\overline{8}$ & $\overline{66.1}$ & $\overline{-}$ & $\overline{-}$ \\
\hline Takao & 79 & $\begin{array}{l}35 \\
44\end{array}$ & $\overrightarrow{2}$ & $\overline{45} \cdot 5$ & $\overline{-}_{1}$ & $\cdots$ \\
\hline
\end{tabular}


Seit in dem Jahre 1896 zuerst in dem Regierungsbezirk Tainan die Pestepidemie herrschte, sah man alljährlich die Pest epidemisch oder sporadisch auftreten. In dieser Zeit wurde vorübergehend ein Quarantäneamt errichtet. Unter den Quarantänebeamten erkrankten (mit Ausnahme der Pestperiode von 1896 und 1900) 7 bis 8 an Pest. In diesem Jahre (1901) wurde das sämtliche Personal, welches zum Quarantänedienst angestellt war, von vornherein geimpft. Die Folge dieser MaBnahme war, daB nur 2 Personen davon erkrankten. In der Tabelle VI werden die Krankheitsund Sterbefälle der Quarantänebeamten im laufenden Jahre in Prozent tabellarisch zusammengestellt.

Tabelle VI.

Krankheitsfälle der Beamten des vorübergehenden Quarantäneamtes im laufenden Jahre:

\begin{tabular}{c||c|c|c}
\hline \hline J a h r & Quarantänebeamte & Krankheitsfälle & Proz. d. Erkrankten \\
\hline 1896 & 68 & - & - \\
1897 & 224 & 2 & 0.09 \\
1898 & 103 & 3 & $0 \cdot 23$ \\
1899 & 272 & 17 & 0.62 \\
1900 & 89 & - & - \\
1901 & 295 & 2 & 0.07
\end{tabular}

Tabelle VII.

Krankheitsfälle der geimpften und ungeimpften Quarantänebeamten in Prozenten im Jahre 1901.

\begin{tabular}{|c|c|c|c|}
\hline & Personen & Krankheitsfälle & Prozente \\
\hline Geimpfte & 258 & 1 & $0 \cdot 04$ \\
\hline Ungeimpfte & 39 & 1 & 0.26 \\
\hline
\end{tabular}

Zum SchluB der Betrachtung der Pestepidemie im Jahre 1901 werden hier verschiedene Fälle in der Tabelle VIII angeführt werden, um die Wirksamkeit des Impfstoffs von Kitasato darzutun.

Tabelle VIII.

Zusammenstellung der bedeutsamsten Fälle, die durch Impfung vor der Pestinfektion geschützt wurden.

Fall 1.

Da am 25. Dezember 1900 ein Gefängnisbeamter, namens T. K. (34 Jahre alt), welcher in der Dienstwohnung der Gefängnisbeamten zu Tainan wohnt, an Pest erkrankt war, wurde am 26. Dezember er und sämtliche Personen 
in dieser Dienstwohnung geimpft; damals waren zwei (S. K., 40 Jahre alt, und K. M., 9 Jahre alt) wegen Abwesenheit ungeimpft geblieben. Am 29. des l. Mon. wurde einer dieser ungeimpft gelassenen S. K. und am 31. der andere K. M. von Pest befallen. Während dieser Zeit hatte der zuerst an Pest erkrankte und durch Impfung geheilte Gefängnisbeamte T. $\mathrm{K}$. in der Wohnung dieser Pestkranken übernachtet, ohne an Pest zu erkranken. Ebenso waren alle Geimpften von der Ansteckung verschont geblieben.

\section{Fall 2.}

Ein Schutzmann J. K. (40 Jahre alt), einer der Bewohner der Dienstwohnung für mehrere Schutzmannfamilien in Anping, erkrankte am 16. Jan. 1901 an Pest, ebenso am 19. sein Sohn M. (16 Jahre alt) und am 26. des 1. Mon. seine Frau T. (22 Jahre alt); alle diese Patienten wurden in das Hospital tür Infektionskrankheiten geschickt. Die sämtlichen Familien in dieser Dienstwohnung waren bereits geimpft worden. Diese erkrankte Familie hat sich vor kurzem, d. h. nachdem bei anderen Familien die Schutzimpfung ausgeführt worden war, von einer anderen Gegend ungeimpft hierher niedergelassen. Deshalb waren alle Familienangehörige infiziert worden, während bei den anderen Familien, die mit der erkrankten stets in Berührung kommen, kein Pestfall zu verzeichnen war.

\section{Fall 3.}

Ein Mann namens T. U. wurde am 12. Januar 1901 geimpft. Er bekam in der Dunkelkeit der Küche von einer mit Pest infizierten Ratte einen $\mathrm{Bi \beta}$ in den FuB, dennoch ist er von der Pest verschont geblieben.

\section{Fall 4.}

In einer Wohnung in Tainan waren alle drei ungeimpfte Familienangehörige an Pest erkrankt, während die Familien der dicht an ihr anschlieBenden beiderseitigen Nachbarwohnungen, wegen ihrer bereits durchgeführten Impfung, von Pest nicht angesteckt waren.

\section{Fall 5.}

Am 8. Januar 1901 wurden alle bei dem Gouvernement zu Tainan dienenden Beamten, Diener usw., sowie alle ihre Familienangehörige und Dienstboten geimpft, die zusammen 342 Personen ausmachen. Ein Hilfsbeamter F. (40 Jahre alt) wurde nach dieser Impfung von dem Mutterlande ungeimpft hierher versetzt. Er erkrankte am 11. April an Pest und starb sofort. Bei anderen Geimpften war keine Spur von Pest nachzuweisen.

\begin{tabular}{|c|c|c|c|c|c|}
\hline Fall & $\begin{array}{l}\text { Familie und } \\
\text { Dienstboten }\end{array}$ & $\begin{array}{c}\text { Geimpfte } \\
\text { Ungeimpfte }\end{array}$ & $\begin{array}{c}\text { Datum der } \\
\text { Impfung }\end{array}$ & $\begin{array}{l}\text { Zahl der } \\
\text { Erkrankten }\end{array}$ & $\begin{array}{c}\text { Beginn der } \\
\text { Krankheit }\end{array}$ \\
\hline 6 & 2 & $\begin{array}{l}1 \\
1\end{array}$ & 5. I. & $\overline{1}$ & 25. I. \\
\hline 7 & 5 & $\begin{array}{l}4 \\
1\end{array}$ & 16. II. & $\overline{1}$ & 21. II. \\
\hline
\end{tabular}


Tabelle VIII. (Fortsetzung.)

\begin{tabular}{|c|c|c|c|c|c|}
\hline Fall & $\begin{array}{l}\text { Familie und } \\
\text { Dienstboten }\end{array}$ & $\begin{array}{l}\text { Geimpfte } \\
\text { Ungeimpfte }\end{array}$ & $\begin{array}{l}\text { Datum der } \\
\text { Impfang }\end{array}$ & $\begin{array}{l}\text { Zahl der } \\
\text { Erkrankten }\end{array}$ & $\begin{array}{l}\text { Beginn der } \\
\text { Krankheit }\end{array}$ \\
\hline 8 & 5 & $\begin{array}{l}4 \\
1\end{array}$ & 15. Ш. & $\overline{1}$ & 9. III.* \\
\hline-9 & 6 & $\begin{array}{l}4 \\
2\end{array}$ & 15. II. & $\overline{1}$ & 16. III. * \\
\hline 10 & 5 & $\begin{array}{l}3 \\
2\end{array}$ & 25. II. & $\overline{1}$ & 15. $\overline{\mathrm{III}}$ * \\
\hline 11 & 4 & $\begin{array}{l}3 \\
1\end{array}$ & 25. II. & $\overline{1}$ & 13. $\overline{\text { III }} *$ \\
\hline 12 & 5 & $\overline{5}$ & $\overline{-}$ & $\overline{2}$ & 13. IV. $13 . \mathrm{IV}$ \\
\hline 13 & 5 & $\begin{array}{l}3 \\
2\end{array}$ & 25. II. & $\overline{1}$ & 15. IV. \\
\hline 14 & 3 & $\begin{array}{l}2 \\
1\end{array}$ & $1.1 \mathrm{~V}$. & $\overline{1}$ & 18. IV. \\
\hline 15 & 5 & $\begin{array}{l}4 \\
1\end{array}$ & 30. IV. & $-\overline{1}$ & 21. IV. \\
\hline 16 & 6 & $\begin{array}{l}5 \\
1\end{array}$ & 27. I. & $\overline{1}$ & 11. IV. \\
\hline 17 & 4 & $\begin{array}{l}1 \\
3\end{array}$ & 4.IV. & $\overline{2}$ & 26. IV. 26.IV. \\
\hline 18 & 4 & $\begin{array}{l}2 \\
2\end{array}$ & 10. I. & $\overline{1}$ & 21. IV. \\
\hline 19 & 5 & $\begin{array}{l}3 \\
2\end{array}$ & 27. IV. & $\overline{1}$ & 28. IV. \\
\hline 20 & 4 & $\frac{2}{2}$ & 7. IV. & $-\overline{1}$ & $29 . \overline{I V}$. \\
\hline 21 & 2 & $\overline{2}$ & $\overline{-}$ & $\overline{2}$ & 29.IV. $29 . I V$. \\
\hline 22 & 2 & $\begin{array}{l}1 \\
1\end{array}$ & 7. IV. & $\overline{1}$ & 30. IV. \\
\hline 23 & 3 & $\begin{array}{l}2 \\
1\end{array}$ & 7. IV. & $\overline{1}$ & $\overline{7 . I V}$. \\
\hline 24 & 4 & $\begin{array}{l}1 \\
3\end{array}$ & 1. IV. & $\overline{2}$ & 23. IV. 23. IV. \\
\hline 25 & 3 & $\begin{array}{l}2 \\
1\end{array}$ & 1. IV. & $\overline{1}$ & 29. IV. \\
\hline 26 & 3 & $\begin{array}{l}1 \\
2\end{array}$ & 1. IV. & $\overline{1}$ & 21. IV. \\
\hline 27 & 4 & $\begin{array}{l}1 \\
3\end{array}$ & 19. IV. & $\overline{2}$ & 27. IV. $30.1 V$. \\
\hline 28 & 3 & $\begin{array}{l}2 \\
1\end{array}$ & 1. IV. & - & 27. IV. \\
\hline 29 & 8 & $\begin{array}{l}4 \\
4\end{array}$ & 2.IV. & $\overline{2}$ & 29. IV. 29. IV. \\
\hline
\end{tabular}


Tabelle VIII. (Fortsetzung.)

\begin{tabular}{|c|c|c|c|c|c|}
\hline Fall & $\begin{array}{l}\text { Familie and } \\
\text { Dienstboten }\end{array}$ & $\begin{array}{c}\text { Geimpfte } \\
\text { Ungeimpfte }\end{array}$ & $\begin{array}{l}\text { Datum der } \\
\text { Impfung }\end{array}$ & $\begin{array}{l}\text { Zahl der } \\
\text { Erkrankten }\end{array}$ & $\begin{array}{l}\text { Beginn der } \\
\text { Krankheit }\end{array}$ \\
\hline 30 & 3 & $\begin{array}{l}2 \\
1\end{array}$ & 28. IV. & $\overline{1}$ & 6. V. \\
\hline 31 & 5 & $\begin{array}{l}4 \\
1\end{array}$ & 11. IV. & $\overline{1}$ & 3. V. \\
\hline 32 & 2 & $\begin{array}{l}1 \\
1\end{array}$ & $\begin{array}{c}\text { 8. IV. } \\
-\end{array}$ & $\overline{1}$ & 6. V. \\
\hline 33 & 2 & $\begin{array}{l}1 \\
1\end{array}$ & 8. IV. & $\overline{1}$ & 2. V. \\
\hline 34 & 3 & $\begin{array}{l}1 \\
2\end{array}$ & 7. IV. & $\overline{2}$ & 5. V. $5 . \mathrm{V}$ \\
\hline $3 \mathfrak{5}$ & 6 & $\begin{array}{l}5 \\
1\end{array}$ & 8. IV. & $\overline{1}$ & $\overline{7 \cdot V}$ \\
\hline 36 & 4 & $\begin{array}{l}2 \\
2\end{array}$ & $\begin{array}{c}\text { 10. IV. } \\
-\end{array}$ & $\overline{1}$ & 7. V. \\
\hline 37 & 2 & $\begin{array}{l}1 \\
1\end{array}$ & 9. IV. & $\overline{1}$ & $10 \overline{\mathrm{V}}$. \\
\hline 38 & 4 & $\begin{array}{l}2 \\
2\end{array}$ & 10. IV. & $\overline{1}$ & $26 . \overline{I V}$. \\
\hline 39 & 7 & $\begin{array}{l}3 \\
4\end{array}$ & 10. IV. & $\overline{4}$ & $\begin{array}{l}\text { 25. IV. } 25 . \text { IV. } \\
\text { 25. IV. 25. IV. }\end{array}$ \\
\hline 40 & 4 & $\begin{array}{l}2 \\
2\end{array}$ & 9. IV. & $\overline{1}$ & 7. V. \\
\hline 41 & 4 & $\begin{array}{l}2 \\
2\end{array}$ & 27. IV. & $\overline{1}$ & 5. V. \\
\hline 42 & 16 & $\begin{array}{l}7 \\
9\end{array}$ & 23. IV. & $\overline{3}$ & 18., 19., 19. IV. \\
\hline 43 & 4 & $\begin{array}{l}3 \\
1\end{array}$ & 9. IV. & $\overline{1}$ & $25 . \overline{I V}$. \\
\hline 44 & 4 & $\begin{array}{l}3 \\
1\end{array}$ & $\begin{array}{l}\text { 7. IV. } \\
-\end{array}$ & $\overline{1}$ & $\overline{1 .} \overline{\mathrm{V}}$ \\
\hline 45 & 6 & 6 & 10. IV. & - & - \\
\hline 46 & 2 & $\begin{array}{l}1 \\
1\end{array}$ & 7. IV. & $-\overline{1}$ & 23. IV. \\
\hline 47 & 4 & $\begin{array}{l}2 \\
2\end{array}$ & 1. IV. & $\overline{1}$ & 27. IV. \\
\hline
\end{tabular}

Anmerkung. 1. Die Fälle 1 bis 5, welche der Eigentümlichkeit der Tatsachen halber nicht tabellarisch abgekürzt werden können, werden einzeln behandelt.

2. Die Fälle von 6 bis 47 fallen in das Jahr 1901.

3. Die bis zur Abfassung dieses Berichtes bekannt gewordenen Todesfälle sind mit * versehen. 
Im nächsten Jahre dieser Pestepidemie (Januar 1902) wurde innerhalb des Gouvernements zu Tainan ein Amt gegen die Pestseuchen errichtet. Die erste Aufgabe dieses Amtes war die möglichst vollständige Vertilgung der Ratten in den Städten Tainan und Anping. Infolgedessen herrschte in den Jahren 1902 bis 1903 keine Epidemie. Als am 23. Februar 1904 in einem Stadtteil in Tainan ein Pestfall ausbrach, verbreitete sich die Seuche mit rapider Geschwindigkeit, so daB die Ausdehnung der Epidemie fast die gleiche wie im Jahre 1901 war. Da also bis jetzt die prärentiven MaBregeln gegen Pest nur minimalen Erfolg hatten, wurde auch diesmal wieder die Schutzimpfung vorgenommen. Am 1. September erlosch die Epidemie.

Die Impfung wurde am 12. April begonnen und hat am 7. Juni geendet. Sie wurde erst in dem Stadtteil, wo die Epidemie am stärksten herrschte, vorgenommen, und dann allmählich in den weniger betroffenen Gegenden. Der Impfstoff ist nach wie vor im Institut für Infektionskrankheiten zu Tokio hergestellt. Es wurde von diesem Impfstoff bei Erwachsenen 1.5 bis 3.0 gebraucht, zur Injektion bei Kindern je nach

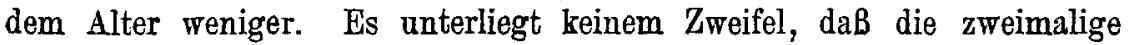
Impfung bedeutende Erfolge erzielt, da aber in Tainan es die Umstände nicht erlaubten, bei einer und derselben Person zweimal zu impfen, so wurde hier nur eine einmalige Impfung ausgeführt.

Da die Art und Weise der Impfung und die anderen Maßnahmen dieses Jahres von dem Jahre 1901 keine Abweichung bieten, so werden hier nur die Resultate der Schutzimpfung tabellarisch aufgestellt. Tainan.

Die Tabelle IX zeigt das Ergebnis der Schutzimpfung der Stadt

$$
\text { Tabelle IX. }
$$

Resultate der Schutzimpfung gegen Pest in Tainan im Epidemiejahre 1904.

\begin{tabular}{c|c|c|c|c|c}
\hline Einwohner & $\left\{\begin{array}{c}\text { Geimpfte } \\
\text { Ungeimpfte }\end{array}\right\} \mid \begin{array}{c}\text { Krankheits- } \\
\text { fälle }\end{array}$ & $\begin{array}{c}\text { Dieselben } \\
\text { in Promille }\end{array}$ & Sterbefälle & $\begin{array}{c}\text { Dieselben } \\
\text { in Promille }\end{array}$ \\
\hline $46264\left\{\begin{array}{c}9906 \\
36358\end{array}\right.$ & $\begin{array}{r}45 \\
1417\end{array}$ & $\begin{array}{c}0 \cdot 5 \\
39 \cdot 0\end{array}$ & $\begin{array}{r}31 \\
1159\end{array}$ & $\begin{array}{r}3 \cdot 1 \\
31.9\end{array}$
\end{tabular}

In der Tabelle $X$ wird das Verhältnis der Krankheits- und Sterbefälle bei den Geimpften in pro Mille zusammengefaßt.

Tabelle X.

Krankheits- und Sterbefälle bei den Geimpften im Epidemiejahre 1904.

\begin{tabular}{c|c|c|c|c}
\hline \hline $\begin{array}{c}\text { Beginn der Krankheit } \\
\text { nach der Impfung }\end{array}$ & $\begin{array}{c}\text { Zahl der } \\
\text { Erkrankten }\end{array}$ & Geheilt & Gestorben & $\begin{array}{c}\text { Sterbefälle } \\
\text { in Promille }\end{array}$ \\
\hline Nach dem 1. Tag & 1 & - & 1 & $100 \cdot 0$ \\
$"$ " 2. " & 6 & 1 & 5 & $833 \cdot 3$ \\
$"$ " 3. " & 2 & - & 2 & $100 \cdot 0$
\end{tabular}


Tabelle X. (Fortsetzung.)

\begin{tabular}{c|c|c|c|c}
\hline \hline $\begin{array}{c}\text { Beginn der Krankheit } \\
\text { nach der Impfung }\end{array}$ & $\begin{array}{c}\text { Zahl der } \\
\text { Erkrankten }\end{array}$ & Geheilt & Gestorben & $\begin{array}{c}\text { Sterbefälle } \\
\text { in Promille }\end{array}$ \\
\hline \hline Nach dem 4. Tag & 1 & - & 1 & $100 \cdot 0$ \\
" " 5. " & 3 & - & 3 & 100.0 \\
über 6. " & 32 & 13 & 19 & $593 \cdot 8$ \\
total & 45 & 14 & 31 & 688.9
\end{tabular}

Da die Sterbefälle bei Ungeimpften 817.92 pro Mille betragen, so vermindern sie sich bei den Geimpften um 129.04 pro Mille. Daß die Sterbefälle nach 6 Tagen bedeutend abnehmen, beweist, daB der Impfstoff, wie Calmette richtig sagt, nach 6 Tagen erst seine volle Wirkung zeigt.

In der Tabelle XI werden, wie in der Tabelle VIII, die bedeutsamsten Fälle in bezug auf die Wirkung der Schutzimpfung zusammengestellt.

Tabelle XI.

Zusammenstellung der verschiedenen Fälle, die durch Schutzimpfung von der Pestansteckung befreit sind (1904).

\begin{tabular}{|c|c|c|c|c|c|c|c|c|c|c|c|}
\hline Fall & 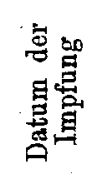 & 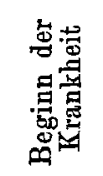 & 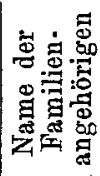 & 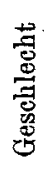 & Alter & Fall & 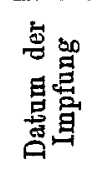 & 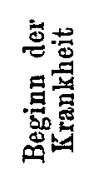 & 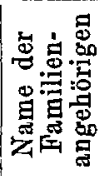 & 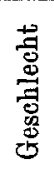 & Alter \\
\hline \multirow[t]{5}{*}{1} & - & 23. V. & K. S. & $\mathrm{m}$. & 71 & \multirow[t]{9}{*}{4} & 9. V. & - & S. K. & m. & 31 \\
\hline & - & 17. VI. & T. T. & w. & 58 & & 15. V. & - & T. T. & $\mathrm{m}$. & 13 \\
\hline & - & 25. V. & Y. S. & w. & 37 & & 15. V. & - & S. K. & w. & 50 \\
\hline & 10. V. & - & G. S. & $\mathrm{m}$. & 9 & & - & - & K. H. & m. & 35 \\
\hline & & & & & & & - & 3. V. & S. H. & w. & 30 \\
\hline \multirow{9}{*}{2} & 28. IV. & - & T. J. & m. & 44 & & - & 6. V. & K. S. & w. & 3 \\
\hline & - & - & T. S. & w. & 2 & & - & 4. V. & S. B. & $\mathrm{m}$. & 2 \\
\hline & - & 6. VI. & T. G. & w. & 8 & & - & 3. V. & F. M. & $\mathrm{m}$. & 19 \\
\hline & 2. VI. & - & T. S. & w. & 5 & & & & $\mathrm{~T}$ & & \\
\hline & - & - & T. S. & w. & 2 & \multirow[t]{5}{*}{5} & 9. V. & - & S. T. & m. & 50 \\
\hline & 一 & 6. VI. & K. R. & m. & 18 & & - & 14. IV. & S. T. & w. & 49 \\
\hline & 2. VI. & - & G. S. & w. & 29 & & 13. V. & - & K. K. & w. & 43 \\
\hline & - & 8. VI. & G. K. & w. & 30 & & - & 22. IV. & G. Z. & w. & 26 \\
\hline & - & - & s, 0 & & 43 & & & & & w. & .10 \\
\hline \multirow[t]{6}{*}{3} & 17. IV. & - & K. B. & w. & 41 & \multirow[t]{2}{*}{6} & $18 . \mathrm{V}$ & - & K. S. & $\mathbf{m}$. & 50 \\
\hline & 15. V. & - & S. R. & w. & 31 & & - & 23. IV. & S. K. & $\mathrm{m}$. & 17 \\
\hline & - & $13 . \mathrm{V}$ & K. K. & m. & 24 & \multirow[t]{4}{*}{7} & - & - & o. s. & m. & 18 \\
\hline & 一 & 23. V. & O. K. & $\mathrm{m}$. & 40 & & 26. IV. & - & O. $\mathbf{T}$. & m. & 14 \\
\hline & & & & & & & - & 14. VI. & O. T. & $\mathbf{m}$. & 8 \\
\hline & & & & & & & - & - & S. S. & w. & 38 \\
\hline
\end{tabular}


Tabelle XI. (Fortsetzung.)

\begin{tabular}{|c|c|c|c|c|c|c|c|c|c|c|c|}
\hline Fall & 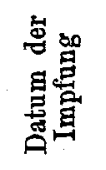 & 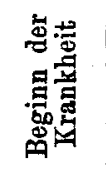 & 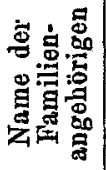 & 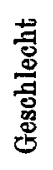 & Alter & Fall & 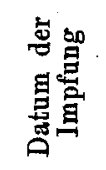 & 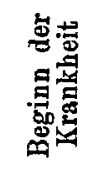 & 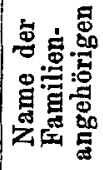 & 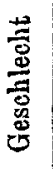 & Alter \\
\hline \multirow[t]{4}{*}{8} & - & - & K. T. & m. & 41 & 15 & 6. VI. & - & H. Y. & m. & 29 \\
\hline & 26. IV. & - & o. T. & w. & 67 & & - & 5. V. & S. K. & w. & 40 \\
\hline & - & 23. V. & T. S. & w. & 35 & & - & 14. IV. & H. T. & m. & 5 \\
\hline & - & 17. V. & T. Z. & $\mathbf{m}$. & 35 & & 6. VI. & - & H. R. & $\mathbf{m}$. & 8 \\
\hline \multirow[t]{6}{*}{9} & 12. V. & - & S. B. & m. & 31 & 16 & - & 2. V. & S. H. & m. & 8 \\
\hline & - & - & K. D. & w. & 28 & & 30. IV. & - & Z. K. & w. & 58 \\
\hline & - & 13. VI. & K. M. & w. & 68 & & - & - & S. K. & w. & 15 \\
\hline & - & 1. V. & S. K. & m. & 14 & & $30.1 \%$ & - & K. K. & m. & 8 \\
\hline & - & 25. V. & K. Y. & w. & 32 & 17 & 17. V. & - & S. $B$. & $\mathrm{m}$. & 34 \\
\hline & & & & & & & - & 2. VI. & S. K. & w. & 8 \\
\hline \multirow[t]{2}{*}{10} & 9. V. & - & S. A. & m. & 25 & & - & 4. VI. & K. S. & w. & 37 \\
\hline & - & 16. V. & R. R. & w. & 15 & & - & 14. IV. & Z. S. & m. & 21 \\
\hline \multirow[t]{4}{*}{11} & 22. IV. & - & S. T. & m. & 12 & 18 & 9. V. & - & o. S. & m. & 44 \\
\hline & - & 17. $\nabla$. & T. S. & w. & 13 & & - & 29. V. & S. Y. & w. & 52 \\
\hline & - & 26. V. & K. K. & w. & 18 & & - & - & O. D. & w. & 17 \\
\hline & & & & & & & 27. IV. & - & K. B. & w. & 35 \\
\hline \multirow[t]{4}{*}{12} & 24. IV. & - & T. S. & m. & 17 & 19 & - & - & Y. S. & m. & 13 \\
\hline & - & 17. IV. & $\begin{array}{l}\text { R. S. } \\
\text { B. }\end{array}$ & w. & $\begin{array}{r}4 \\
43\end{array}$ & & 27. IV. & - & S. S. & w. & 54 \\
\hline & - & $\overline{\mathrm{V}}$ & $\begin{array}{l}n_{0} \\
R\end{array}$ & w. & $\begin{array}{r}40 \\
4\end{array}$ & & - & 24. V. & S. K. & w. & 13 \\
\hline & & & & & & & - & 27. V. & Y. Y. & m. & 15 \\
\hline \multirow[t]{7}{*}{13} & 24. IV. & - & R. T'. & m. & 51 & & 27. IV. & - & K. S. & m. & 45 \\
\hline & 12. V. & - & K. R. & w. & 38 & 20 & 30. IV. & - & G. S. & $\mathbf{m}$. & 15 \\
\hline & - & - & R. T. & m. & 23 & & - & - & K. B. & w. & 12 \\
\hline & - & 13.VII. & S. G. & w. & 18 & & - & 21. V. & K. H. & w. & 17 \\
\hline & 12. V. & - & G. D. & w. & 80 & & - & 1. V. & G. S. & w. & 28 \\
\hline & 12. V. & - & T. D. & $\mathrm{m}$. & 9 & 21 & 17. V. & - & S. B. & $\mathrm{m}$. & 40 \\
\hline & 12. V. & - & R. M. & $\mathrm{m}$. & 9 & & - & 16. V. & s. s. & $\mathrm{m}$. & 34 \\
\hline \multirow[t]{5}{*}{14} & - & - & S. B. & $\mathrm{m}$. & 50 & & 一 & 16. V. & o. K. & w. & 26 \\
\hline & - & - & K. $\mathbf{S}$. & w. & 30 & & 一 & 16. V. & S. R. & $\mathrm{m}$. & 41 \\
\hline & 26. IV. & - & R. K. & w. & 26 & 22 & - & - & S. R. & m. & 39 \\
\hline & - & 29. IV. & R. K. & m. & 13 & & - & 12. V. & B. R. & w. & 69 \\
\hline & 24. IV. & - & B. $\mathrm{K}$. & m. & 16 & & $\begin{array}{l}\text { 13. V. } \\
13 . \mathrm{V} .\end{array}$ & - & $\begin{array}{l}\text { S. K. } \\
\text { S. T. }\end{array}$ & $\begin{array}{l}\mathrm{m} . \\
\mathrm{m} .\end{array}$ & $\begin{array}{l}8 \\
5\end{array}$ \\
\hline
\end{tabular}

